\title{
Current and emerging biologics for the treatment of hereditary angioedema
}

\author{
Francesca Perego ${ }^{a}$, Maddalena A. Wu ${ }^{b, c}$, Anna Valerievad ${ }^{d}$, Sonia Cacciab ${ }^{b}$ Chiara Suffritti ${ }^{b}$, Andrea Zanichelli ${ }^{b, c}$, \\ Luigi Bergamaschini ${ }^{\mathrm{b}, \mathrm{e}}$ and Marco Cicardib,c
}

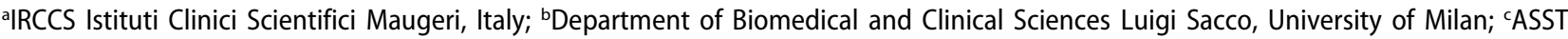
Fatebenefratelli Sacco, Milan, Italy; ${ }^{d C}$ Clinical Centre of Allergology, University Hospital "Alexandrovska", Medical University of Sofia, Bulgaria; ePio Albergo Trivulzio, Milan, Italy

\section{ABSTRACT}

Introduction: Hereditary angioedema due to C1-INH deficiency (C1-INH-HAE) is a rare disease with unpredictable, self-limiting and localized swelling episodes involving the cutaneous and subcutaneous tissues. In the last decade, the spectrum of the possibilities to control the disease has considerably changed with the development of biologic therapies making necessary a careful evaluation of the differences among current and emerging treatments to properly optimize the management of patients. Areas covered: This review serves to summarize the literature regarding the use of biologics for the treatment of C1-INH-HAE. Medications already available on the market and new drugs in different phases of development are addressed.

Expert opinion: the advent of biologic therapies dramatically improved the lives of patients with C1INH-HAE although further improvement is still needed. Whether this is cost/effective will be answered in the next years when we will see if these major advances will benefit the majority of the patients.
ARTICLE HISTORY

Received 16 December 2018 Accepted 12 March 2019

\section{KEYWORDS}

Hereditary angioedema; rare diseases; biologics; treatment; clinical trial: chronic disease

\section{Introduction}

Increasing knowledge on disease pathophysiology allows developing specific 'biologic therapies'. More or less restrictive definitions of biotherapeutics can be found. Here we consider as biologic therapies those treatments that specifically target a biologic function. These types of biologics can be extracted from living organisms as plasma-derived products, produced from specifically modified organisms, as recombinant products and monoclonal antibodies, but also synthetized to interfere with enzymatic function as well as to modify protein synthesis.

\subsection{Biologic therapies}

C1 inhibitor deficiency (C1-INH-HAE) is characterized by episodes of angioedema that last $2-5$ days and resolves spontaneously. Outside from these episodes, clinical abnormalities are not apparent. Thus, treatment of HAE patients can be addressed to revert attacks (on-demand treatment, ODT) or to prevent recurrences (long-term prophylaxis treatment, LTP). Prophylaxis can also be used to prevent symptoms in conditions at high risk as surgical procedures (short-term prophylaxis, STP). Several drugs are already on the market for these purposes and others are under investigation. Available treatments prevent disease mortality and reduce disease burden. However, the high variability of the disease calls for further development to treat affected subjects safely and precisely.

In 1963, Donaldson et al. [1] discovered C1 inhibitor deficiency as genetic defect causing HAE and shortly thereafter, this protein, extracted from human plasma, became the first therapeutic options for these patients. Evolving from the original plasma product $\mathrm{C} 1-\mathrm{INH}$ has been adapted for subcutaneous administration to facilitate replacement therapy for attack prophylaxis. The same protein is now available as recombinant product from transgenic rabbits.

When it became clear that bradykinin was the principal mediator of angioedema symptoms in C1-INH deficiency, therapies started to be designed to targeted bradykinin production/activity. This category of pharmaceuticals now includes the antagonist of the bradykinin B2 receptor (BK B2R) icatibant; ecallantide, a recombinant selective inhibitor of the bradykinin releasing enzyme plasma kallikrein (pK); the anti-pK monoclonal antibody lanadelumab; the small pK inhibitory molecule, BCX 7353.

It is also clear, now, that Factor XII (FXII or Hageman factor) has a central role in activating the kallikrein-kinin system, which produces bradykinin $[2,3]$. This prompted the development of monoclonal antibodies to block activated Factor XII (FXIla).

A number of therapeutic options targeted to block bradykinin are now available or under development for HAE (Tables 1 and 2). The availability of the treatment is different by country according to the local regulation [4]. These products reach the target using different mechanisms (Figure 1). The variety of interventions let envisage a future optimal tailoring of the treatments to different phenotypes and personal needs [5]. Here we will review the characteristics of these therapeutics. 
Article highlights

- The spectrum of biologic therapies has dramatically widened over the last decades, paralleling the progress of biotherapeutics in other fields, such as autoimmune and neoplastic diseases.

- Current biologic treatment options for hereditary angioedema include plasma-derived and recombinant $\mathrm{C} 1-\mathrm{INH}$, recombinant new proteins and monoclonal antibodies (as ecallantide, lanadelumab and monoclonal antibodies blocking FXIla) as well as synthetic peptides and small molecules (as icatibant, BCX 7353 and KVD900).

- The therapeutic options for children have recently widened and include not only pdC1-INH but also icatibant.

- Among emerging promising treatments is also gene therapy, namely SERPING1 replacement and antisense oligonucleotide inhibiting prekallikrein.

- Possible limitations to the use of biologic therapies are due to the risk of immunogenicity (even though rare) and to direct-indirect costs (which might be dampened in the long term, also thanks to selfadministration).

This box summarizes key points contained in the article.

\section{Plasma-derived and recombinant $\mathrm{C} 1$ inhibitors}

\subsection{Plasma-derived C1-INH}

The first C1-inhibitor concentrate purified from pooled human plasma dates back to 1974, prepared by the Central Laboratory of the Netherlands Red Cross Blood Transfusion Service (now Sanquin Blood Supply Foundation) [6]. Marketing authorizations in Europe started in 1985, while in the U.S., it became available only in 2008.

In Italy, between 1979 and 1985, a C1-INH preparation from Immuno Vienna was available, for compassionate use to treat severe life-threatening attacks in HAE patients. No virucidal measures were available at that time for plasma products, and wide transmission of hepatitis C virus (86\% of exposed subjects), but no HIV, was associated to this preparation [6]. Starting from the eighties, policies for donors' selection and the introduction of specific manufacturing processes, drastically reduced the risk of viral infection from blood products. Since 1985, different plasmaderived $\mathrm{C} 1-\mathrm{INH}$ (pdC1-INH) preparations were registered in Europe for acute treatment of HAE attacks, and no further evidence of infection related to these preparations has ever been reported [6-9]. Despite the extended use of pdC1-INH, the first registrative study was published in 2009 [7]. It was a randomized, placebo-controlled, double-blind study that compared the efficacy of pasteurized $\mathrm{C} 1$ esterase inhibitor concentrate (Berinert ${ }^{\oplus}$, CSL Behring) with placebo in the treatment of single, acute abdominal or facial attacks in patients with HAE. This trial demonstrated that the median time to onset of symptom relief was significantly shorter with $\mathrm{C} 1-\mathrm{INH} 20 \mathrm{U} / \mathrm{kg}(0.5 \mathrm{~h})$ than with placebo $(1.5 \mathrm{~h} ; \mathrm{p}<.005)$. Moreover, safety and tolerability of $\mathrm{pdC} 1-\mathrm{INH}$ were confirmed and no seroconversions were observed for HIV, hepatitis virus or human B19 virus. According to the results of the above-mentioned trial, the posology of pdC1-INH concentrate is based upon weight, with 20 units $/ \mathrm{kg}$ body weight given as slow injection (5-10 $\mathrm{min}$ ) through a peripheral vein. Most patients undergo resolution of the attack after the first ODT and fewer than $5 \%$ of the attacks require a second administration [10]. Pharmacokinetic parameters were calculated in HAE patients receiving intravenous injections of $\mathrm{C} 1-\mathrm{INH}$ concentrate in an

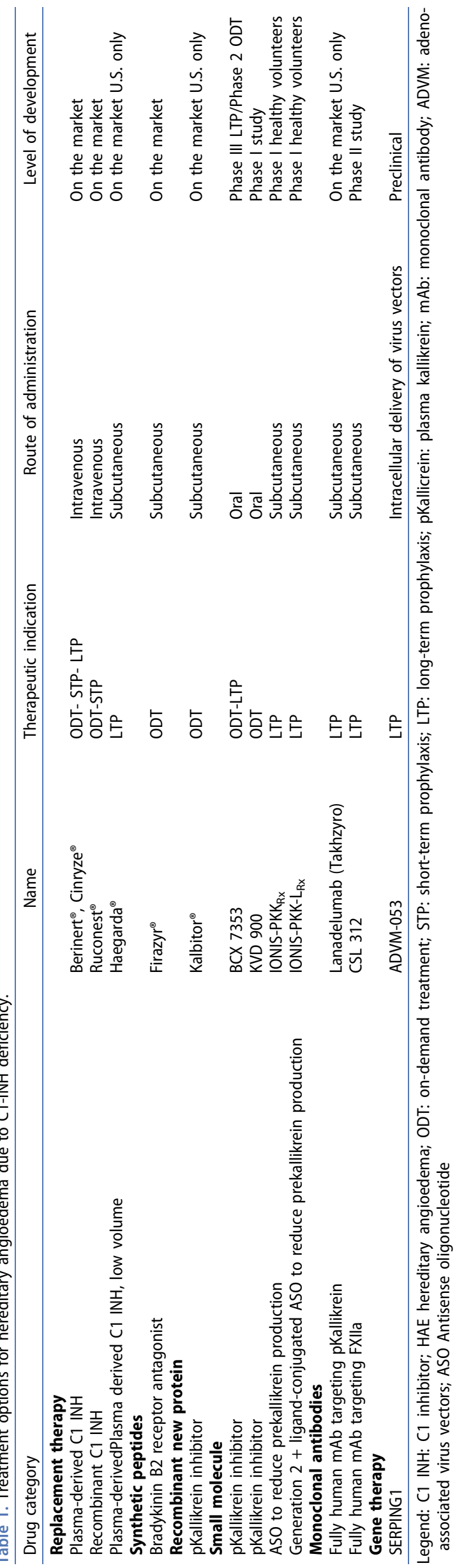


attack-free interval: the elimination half-life $(\mathrm{t} t / 2)$ was $33.3 \pm 19.8 \mathrm{~h}$. Another preparation of plasma-derived, nanofiltered $\mathrm{C} 1-\mathrm{INH}$ has also been shown to be effective for acute treatment of HAE [11]. This study also tested efficacy in preventing attacks and showed that $1000 \mathrm{U}$ given i.v. every three to four days resulted in 50\% reduction of attack rate and significant decrease in severity and duration of breakthrough attacks. An open-label multicenter extension study enrolling 146 C1-INH-HAE subjects confirmed initial data showing that treatment optimization needs individual dose adjustment [12]. A study evaluating escalating doses of Cinryze ${ }^{\oplus}$ prophylaxis revealed that up to $2500 \mathrm{U}$ this product was well tolerated and reduced attack frequency in the majority of patients [13].

It is largely documented that efficacy of all ODTs is maximized by early administration [14-16]. Thus, patient is encouraged and educated to self-administer on-demand therapy at attack onset.

For STP $1000 \mathrm{U}$ Cinryze ${ }^{\circledR}$ should be given within $24 \mathrm{~h}$ of the procedure and $1000 \mathrm{U}$ Berinert ${ }^{\circledR}$ within $6 \mathrm{~h}$ of the procedure, being administration close to or immediately before the procedure a reasonable choice.

PdC1-INH is treatment of choice for ODT, STP, and LTP for pregnant women [17].

Before the recent phase 3 multicenter trial demonstrating the efficacy and safety of icatibant also for the pediatric population [18-20], pdC1-INH was the only available drug for treatment of acute attacks and STP (and seldom LTP) in children with C1-INH-HAE $[10,21]$.

Cumulative experience in clinical trials and in practice, makes evident that pdC1-INH has an excellent safety profile. Reported adverse effects are rare and mild, including headache and fever, together with unusual allergic reactions characterized by edema, urticaria, itching and, in a very few circumstances, anaphylactic shock [22].

During the first 3 years in the U.S. market, 10 serious thrombotic events were reported as associated to i.v. Cinryze $^{\circledast}$ given for LTP [23]. Those thrombotic events were ascribed to indwelling devices, as port-a-cath, for i.v. delivery and not to the drug itself.

So far, there is no evidence that repeated use of pdC1-INH can induce a resistance towards the drug.

In 2014 a prospective, randomized, open-label, crossover study was conducted by Martinez-Saguer et al. to evaluate the pharmacokinetics of the i.v. formulation of pdC1-INH after subcutaneous versus intravenous administration in subjects with mild or moderate HAE during an attack-free interval [24]. The study demonstrated that the mean relative bioavailability of functional C1-INH after subcutaneous administration was almost $39.7 \%$ and $\mathrm{C} 1-\mathrm{INH}$ antigen as well as C4 antigen levels were comparable to C1-INH activity levels in terms of relationship between the two routes of administration.

A formulation of pdC1-INH (CSL830), volume-reduced (500 $\mathrm{U} / \mathrm{mL}$ ) for subcutaneous use, was tested in the COMPACT phase II trial, demonstrating to be well tolerated and able to provide a dose-dependent increase in physiologically relevant functional C1-INH plasma levels [25].

The subsequent phase III study, evaluated twice-weekly s.c. administration of either $40 \mathrm{IU} / \mathrm{kg}$ or $60 \mathrm{IU} / \mathrm{kg}$ in leading. Along with the endpoints of clinical efficacy, the trial tested the 


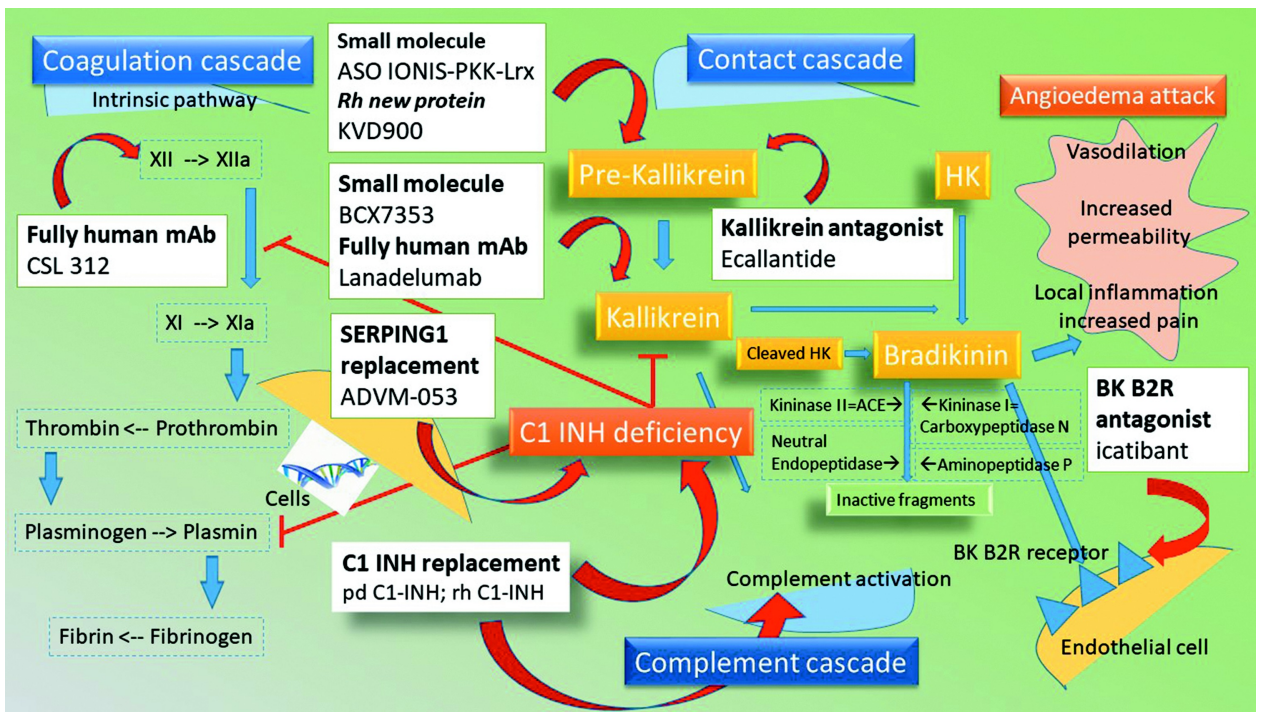

Figure 1. Site of action of current and emerging biologics for the treatment of hereditary angioedema.

INH: inhibitor; pd-: plasma derived; rh: recombinant; mAb: monoclonal antibody; ADVM: adeno-associated virus vectors; ASO IONIS-PKK Antisense oligonucleotide: ASO inhibiting Prekallikrein; BK B2R: bradykinin beta2 receptor; ACE: angiotensin-converting enzyme. The white squares contain the treatment options. The red arrows indicate the site of action of each medication.

capacity of this treatment to maintain patients on $\mathrm{C} 1-\mathrm{INH}$ functional plasma levels of $40 \%$ of normal value, a level predicted to be protective from angioedema attacks. The number of attacks per month was 1.2 in the $40 \mathrm{U} / \mathrm{Kg}$ group, 0.5 in the $60 \mathrm{U} / \mathrm{Kg}$ and 4.0 in the placebo. C1-INH plasma levels reached a steady state after three weeks of treatment. At $60 \mathrm{U} / \mathrm{Kg}$ mean $\mathrm{C} 1-\mathrm{INH}$ function in plasma was $48 \%$ (range 38-60) with dose-dependent reduction in attack risk [26-28]. FDA approved $60 \mathrm{U} / \mathrm{Kg}$ of subcutaneous C1-INH (Haegarda ${ }^{\circledR}$ ) twice per week for prophylaxis of attacks in patients with HAE due to C1-INH deficiency.

\subsection{Recombinant human $\mathrm{C} 1-\mathrm{INH}$}

Recombinant human $\mathrm{C} 1$ inhibitor (rhC1-INH, Conestat alpha, Ruconest $^{\oplus}$, Pharming Technologies B.V., Leiden, The Netherlands) is obtained through a purification process of transgenic New Zealand white rabbits' milk. The drug has been evaluated for its efficacy for treatment of $\mathrm{C} 1$-INH-HAE patients during acute angioedema attacks in several studies, including three double-blind, placebo-controlled efficacy studies [29,30] and five open-label studies [31-35]. Treatment resulted in rapid attack resolution and no relapses or rebound suggesting, that despite a shorter half-life (approximately $2 \mathrm{~h}$ ), rhC1-INH has an activity comparable to that of the plasma products. A meta-analysis of trials using pd- or rhC1INH showed that efficacy mainly relays on the infused amounts and not on the pharmacokinetic characteristics.

Accordingly, one open-label and one double-blind, randomized, placebo-controlled study showed that $\mathrm{rhC} 1-\mathrm{INH}$ is effective in preventing attacks when given intravenously once or twice per week $[35,36]$. The mean number of attacks of HAE over 4 weeks was significantly reduced with $\mathrm{rhC} 1-\mathrm{INH}$ twice weekly, and once weekly, versus placebo, with mean differences of -4.4 attacks and -2.8 attacks, respectively [36].
In addition, small case report series confirmed efficacy in LTP and in STP before deemed invasive medical procedures [37].

The development plan of the company indicates new approaches for subcutaneous, intramuscular and transdermal patch delivery [https://www.pharming.com/release-annualreport-2017/]. The safety data analyses about rhC1-INH demonstrate that the drug is generally safe and well tolerated when administered for treatment and prevention of $\mathrm{HAE}$ attacks. The adverse event profile found in the randomized, placebo-controlled studies was similar for patients treated in the rhC1-INH and placebo treatment groups. There was no increase in the incidence of treatment-emergent adverse events with higher rhC1-INH dose (50 and $100 \mathrm{IU} / \mathrm{kg}$ ), administration of additional rhC1-INH doses for an attack, or with repeated treatment of subsequent attacks [31]. The most common adverse reactions $(\geq 2 \%)$ reported in all clinical trials were headache, nausea, and diarrhea.

Although the drug is considered generally safe, important for clinical practice is to avoid its use in patients who have rabbit allergy due to potentially serious allergic reactions, which has happened within $3 \mathrm{~min}$ after administration of $\mathrm{rhC1}-\mathrm{INH}$ in a single healthy volunteer with a pre-existent (retrospectively known), non-disclosed rabbit dander allergy [38].

Immunogenicity of rhC1-INH was extensively tested throughout the clinical development programs, supporting that rhC1-INH has low potential to induce anti-C1-INH antibodies or anti-host-related impurities (HRI) response. Confirmed antibodies against C1-INH or HRI were observed infrequently and were not associated with clinical symptoms indicating hypersensitivity or changes in rhC1-INH efficacy [39]. There was no association between treatment-emergent adverse events or new acute HAE attacks and the presence of any confirmed anti-C1-INH or anti-HRI antibodies.

No anti-rabbit lgE antibodies were reported elevated with treatment with $\mathrm{rhC} 1-\mathrm{INH}$, and no severe hypersensitivity reactions 
were reported beyond the single case in the phase 1 study, and in the post-approval phase.

\section{Recombinant new protein and monoclonal antibodies}

\subsection{Ecallantide for ODT}

Ecallantide (Kalbitor ${ }^{\circledR}$, Dyax, Cambridge, MA) is a potent and specific inhibitor of plasma kallikrein. It is a recombinant 60amino acid long protein synthesized in the yeast Pichia pastoris [40-43]. Development studies (EDEMA0, EDEMA1, EDEMA2, EDEMA3 - double-blind and repeat dosing, EDEMA4, and DX-88/19) investigated the use of ecallantide in patients with hereditary angioedema $[40,41,44]$. The results for $30 \mathrm{mg}$ subcutaneous injection of ecallantide were superior to placebo and provided sustained relief up to $24 \mathrm{~h}$ after treatment $[40,41]$ when administered within $8 \mathrm{~h}$ of onset of a moderate to severe HAE attack in patients $\geq 10$ years of age. It is now registered in U.S. for acute HAE manifestations in patients 12 years of age, or older, with a dose of $30 \mathrm{mg}$ (3 separate vials of $10 \mathrm{mg}, 1 \mathrm{ml}$ ) for subcutaneous administration [Kalbitor (ecallantide) - short product characteristic of the drug]. The use of the drug is associated with hypersensitivity reactions in up to $10.8 \%$ of the cases $(8.7 \%$ when administered subcutaneously and $17.9 \%$ intravenously). These hypersensitivity reactions fulfill the criteria for systemic hypersensitivity (type 1 anaphylactic or anaphylactoid reactions), in up to $4.4 \%$ of the cases symptoms began within $1 \mathrm{~h}$ of drug exposure. Due to hypersensitivity reactions in the development studies, the drug is to be administered by a health-care professional experienced to treat both anaphylaxis and HAE.

\subsection{Lanadelumab for LTP}

Lanadelumab (DX-2930) is a recombinant, Chinese hamster ovary cell-expressed, fully human IgG1, kelight-chain, monoclonal antibody that was discovered by screening an antibody phage display library21 against purified pK $[45,46]$.

It is a potent (Ki $1 / 4125 \mathrm{pM}$ ) and specific inhibitor of pK. As for other monoclonal antibodies, the pharmacokinetic profile allows one subcutaneous injection of lanaldelumab to inhibit pK for at least two weeks. Lanadelumab was first evaluated in a single-center, double-blinded study in 32 healthy subjects randomized to receive a single subcutaneous administration of the drugs [45]. In this first evaluation, no dose-limiting toxicity was registered. Major and commonly reported adverse events were headache. At different doses (0.1-, 0.3-, 1.0-, and 3.0-mg/kg dose groups) mean elimination half-lives were 20.6, 16.8, 17.6, and 21.2 days, respectively. Dose- and timedependent inhibition of pK was evident.

After this preliminary data a phase $1 \mathrm{~b}$, multicenter, doubleblind, placebo-controlled, multiple-ascending-dose trial was conducted [47]. Thirty-seven patients with HAE due to C1INH deficiency were randomly assigned in a 2:1 ratio to receive either lanadelumab (24 patients) or placebo (13 patients), in two administrations 14 days apart and in different dose groups (30 mg, $100 \mathrm{mg}, 300 \mathrm{mg}$ or $400 \mathrm{mg}$ ). The efficacy measure was the rate of attacks of angioedema within the period of 8-50 days after administration of the treatment compared with the placebo group.

Most common adverse events, after injection site pain, were headache, the mean elimination half-life was 2 weeks. Lanadelumab was completely effective in resetting the number of attacks in the totality of patients in the 300-mg dose group and in $82 \%$ of the $400-\mathrm{mg}$ dose group compared to placebo. The pivotal phase III, multicenter, randomized, double-blind, placebo-controlled study [HELP Study ClinicalTrials.gov. Efficacy and Safety Study of DX-2930 to Prevent Acute Angioedema Attacks in Patients With Type I and Type II HAE. https://clinical trials.gov/ct2/show/NCT02586805. Accessed 10 February 2017] enrolled up to 120 patients to further assess the safety and efficacy of lanadelumab to prevent acute angioedema attacks and now it has been published [48]. Patients were treated for 26 weeks with subcutaneous lanadelumab at 4 different regimens: $150 \mathrm{mg}$ every 4 weeks, $300 \mathrm{mg}$ every 4 weeks, 300 mg every 2 weeks, or placebo. All patients received injections every 2 weeks, the primary efficacy end point was the number of attacks. Compared with placebo, the most effective treatment regimen was 300 mg every 2 weeks with a difference in the attack rate per month of $-1.71(P<001)$ compared with placebo.

Patients who completed the double-blind study were continued into the long-term open-label extension that enrolled additional 100 new patients [HELP Study Extension ClinicalTrials.gov. Long-term Safety and Efficacy Study of DX-2930 to Prevent Acute Angioedema Attacks in Patients With Type I and Type II HAE. https://clinicaltrials. gov/ct2/show/NCT02741596. Accessed 10 February 2017] [49]. Lanadelumab is now approved in U.S. E.U. for HAE LPT at doses of $300 \mathrm{mg}$ every two weeks.

\subsection{Monoclonal antibodies blocking factor XIII}

The fully human anti-FXIla monoclonal antibodies (mAb) $3 F 7$ is a potent and highly specific inhibitor of the proteolytic activity of FXIla. 3F7 binds to rabbit, mouse and human activated FXII. Administration of $3 F 7$ abrogated skin edema induced by contact activation triggered by mast-cell released heparin in mice. 3F7 was also shown to abolish bradykinin-mediated increase of vascular permeability induced by the angiotensin-converting enzyme (ACE) inhibitor captopril in C1-inhibitor-deficient mice. Comparison of $3 F 7$ with current HAE therapeutics in these murine edema models revealed that $3 \mathrm{~F} 7$ has potent and prolonged efficacy. CSL312, a variant of 3F7 with improved affinity and potency, effectively inhibited dextran sulfate triggered FXII contact activation and bradykinin formation in plasma of healthy donors and HAE patients. FXIla, besides cleaving FXI and inducing fibrin production, cleaves plasma prekallikrein to $\mathrm{pK}$, which in turn cleaves high molecular weight kininogen to release bradykinin [Zhihui (Helen) Cao, Biondo M, Rayzman V et al. Development and Characterization of an Anti-FXIla Monoclonal Antibody for the Treatment of Hereditary Angioedema. J Allergy Clin Immunol Feb 2015135 (2S), Page AB194 Abstract].

A Multicenter, Randomized, Placebo-controlled, Parallelarm Study to Investigate the Efficacy, Pharmacokinetics, and Safety of CSL312 in subjects with HAE will start recruitment soon [https://www.clinicaltrials.gov/ct2/show/NCT03712228? term $=$ csl312\&rank $=1]$. 


\section{Synthetic peptides and small molecules}

\subsection{Icatibant}

Icatibant, previously designated as $\mathrm{HOE} 140$, is a synthetic peptide highly specific antagonist for BK-B2R. It was shown to inhibit bradykinin-induced vasodilation in humans. Furthermore, increased vascular permeability in C1-INH knockout mice was reversed by using icatibant. A phase II study first demonstrated the clinical usefulness of antagonizing bradykinin binding to BK-B2R in HAE [50]. It is approved for treatment of attacks in HAE patients since 2008 in Europe and since 2011 in the U.S.

The efficacy (e.g. reduction in symptom severity, onset of primary symptom relief and complete relief) and safety of icatibant for treatment of acute angioedema attacks was demonstrated by three double-blind, randomized, multicenter trials, comparing icatibant versus either placebo or tranexamic acid [51-53]. A recent phase III, multicenter, open-label, nonrandomized, single-arm study showed that icatibant is safe, effective and well tolerated as ODT in the pediatric population (children and adolescents aged 2 years to less than 18 years) [18,54].

Icatibant is dispensed in pre-filled syringes of $3 \mathrm{ml}$ containing $30 \mathrm{mg}$ of the medication, which must be administered by slow subcutaneous injection, preferably in the abdominal region. Icatibant is indicated only for on-demand therapy of acute HAE attacks in adults, adolescents, and children aged 2 years and older. The half-life is about 1-2 $\mathrm{h}$.

Even though a single injection of icatibant is usually sufficient to achieve full recovery, a second injection can be administered after $6 \mathrm{~h}$ if needed (and a third injection may follow after 6 additional hours, without exceeding the dose of three injections per day).

In children and adolescents the recommended dose of icatibant is based on body weight (10 mg for children from $12 \mathrm{~kg}$ to $25 \mathrm{~kg}$ body weight, $15 \mathrm{mg}$ from $26 \mathrm{~kg}$ to $40 \mathrm{~kg}, 20 \mathrm{mg}$ from $41 \mathrm{~kg}$ to $50 \mathrm{~kg}, 25 \mathrm{mg}$ from $51 \mathrm{~kg}$ to $65 \mathrm{~kg}, 30 \mathrm{mg}$ over $65 \mathrm{~kg}$ ). The safety and efficacy of icatibant have not been established yet in children under 2 years of age or weighing less than $12 \mathrm{~kg}$. The subcutaneous route of administration facilitates home treatment preventing vein access problems, often present in pediatric age [55].

The most common adverse reaction to icatibant is mild transient erythema and pain at the injection site. Gastrointestinal complaints (abdominal pain, nausea), headache, fever, asthenia, dizziness, and increase in transaminases have been rarely reported in patients using icatibant without prove of being drug related. Icatibant is contraindicated during acute ischemic events to avoid inhibition of bradykinin vasodilatory effect.

\section{2. $B C X 7353$}

It is a potent synthetic small molecule designed to block the enzymatic site of human plasma kallikrein activity. BCX7353 potently inhibits kallikrein activity, cleavage of $\mathrm{HK}$ in normal and HAE plasma, and suppresses the release of bradykinin after contact system activation on endothelial cells. In vitro potency and specificity of $\mathrm{BCX} 7353$ for plasma kallikrein leads also to a reduction in potency of FXIla relative to $\mathrm{IC}_{50}$ for $\mathrm{pK}$ interfering with the coagulation cascade due to its steric analogy between the two enzymes [Chen X, Kotian P, Wilson R. Preclinical Characterization of BCX7353, an Oral Plasma Kallikrein Inhibitor, for the Treatment of Hereditary Angioedema. AAAAI Congress 2017 Abstract]

The first phase I study [Cornpropst M, Dobo S, Collier J, et al. $B C X 7353$, a potent inhibitor of plasma kallikrein, shows sustained maximal enzyme inhibition when dosed orally once daily: results from a phase 1 trial in healthy subjects. J Allergy Clin Immunol 2016; 137: AB401. abstract] conducted in 92 human healthy volunteers, evaluated the pharmacokinetics, pharmacodynamics, and safety of this pK inhibitor. Each healthy subjects received single $(10,30,100,250,500$ or $1000 \mathrm{mg})$ or multiple $(125,250,500 \mathrm{mg} \mathrm{x} 7$ days or $350 \mathrm{mg}$ $x 14$ days), once-daily (QD) oral doses of $B C X 7353$ or placebo. The half-life of BCX7353 was 50-60 h and, kallikrein inhibition was highly correlated to plasma concentrations. The adverse events reported were gastrointestinal and maculopapular rash demonstrating a good safety profile. The molecule was tested for its efficacy in the reduction of the number of angioedema attacks in an international, three-part, dose-ranging, placebocontrolled trial, where four doses of BCX7353 $(62.5 \mathrm{mg}$, $125 \mathrm{mg}, 250 \mathrm{mg}$, and $350 \mathrm{mg}$ once daily) for the prevention of angioedema attacks over a 28-day period were evaluated [56]. In the 72 of the 77 patients who completed the trial the rate of confirmed angioedema attacks was significantly lower among patients who received $\mathrm{BCX} 7353$ at daily doses of $125 \mathrm{mg}$ or more than among those who received placebo with confirmed mild gastrointestinal symptoms as the principal side effect. (Funded by BioCryst Pharmaceuticals; APeX-1 ClinicalTrials.gov number, NCT02870972.). A phase III randomized, double-blind, placebo-controlled, three-arm trial testing two doses of BCX7353 (110 mg and $150 \mathrm{mg}$ ) for prevention of angioedema attacks, started in march 2018 (APeX-2). The trial consider as a primary efficacy endpoint the rate of angioedema attacks over 24 weeks of study drug administration (ClinicalTrials.gov Identifier: NCT03485911)

The molecule was also tested for the treatment of acute attack at a dosage of $750 \mathrm{mg}$. Data from the preliminary phase of the study [https://www.epgonline.org/uk/news/initialresults-from-zenith-1-trial-of-bcx-7353-or-both-prophylacticand-acute-treatment-of-hae-attacks-biocryst-pharma-.html Data from Pharmawand - Curated by EPG Health - Date added 6 September 2018] showed that patients who selftreated their HAE attacks on a blinded basis with oral BCX 7353 or oral placebo had improvement in symptoms and differences of the VAS scores as early as $1 \mathrm{~h}$ after oral BCX 7353 dosing, and were sustained through $24 \mathrm{~h}$ highlighting an attractive profile also for patients seeking an oral treatment for acute HAE attacks.

\subsection{KVD900}

KVD 900 (KalVista) is a novel small molecule, selective and orally available pK inhibitor that is protective against pKmediated high molecular weight kininogen (HK) cleavage in undiluted HAE and control plasma in an ex vivo assay. KVD900 
protects $\mathrm{HK}$ from ex vivo pK mediated cleavage in plasma from patients with HAE [Feener EP, Murugesan N, Robson PA et al. Results from a semi-automated capillary-based immunoassay. EAACI 26 May 2018-30 Munich]. KVD900 is under investigation in a phase I trial in healthy volunteers to evaluate its safety and tolerability [https://www.clinical-trials.gov]. Preliminary data suggest that KVD900 prevented the breakdown of kininogen in a dose-dependent manner in both HAE patients and in healthy volunteers and it was shown to be generally well tolerated at doses as high as $600 \mathrm{mg}$. It exhibits high solubility and high permeability with rapid uptake into the plasma and high plasma concentrations, these characteristics suites for a new ODT of acute HAE attacks in the next future. A phase II clinical trial for KVD900 is currently planned to start in late 2018 and to be completed in mid-2019 [http:// ir.kalvista.com/node/7656/html].

\section{Gene therapy}

\subsection{SERPING1 supplementation}

Preclinical studies on the use of gene supplementation therapy to cure $\mathrm{C} 1-\mathrm{INH}-\mathrm{HAE}$ provided promising results in a $\mathrm{C} 1-\mathrm{INH}$ deficient murine model presenting characteristics associated with HAE in humans [57]. Single systemic administration of extrachromosomal copies of the human C1-INH gene (SERPING1) into the mice cells through a transfer vector resulted in persistent human $\mathrm{C} 1-\mathrm{INH}$ plasmatic function and protection against increased vascular permeability. The long-term gene expression coupled to leakage relief suggests that long-lasting protective effect and, that this delivery system could offer long-term protection to individuals affected by C1-INH-HAE following a single administration.

In patients, efficacy and safety of gene therapy with adenoassociated virus (AAV) vectors have been demonstrated for both hemophilia $B[58,59]$ and hemophilia $A[60]$, maintaining protective levels of FIX and FVIII, respectively.

The challenge that has to be addressed in HAE individuals is to restore $\mathrm{C} 1-\mathrm{INH}$ synthesis in cells that already produce an altered form of $\mathrm{C} 1-\mathrm{INH}$, which could potentially impair the expression of the wild-type one. The phase I/II advanced clinical trial, has now been discontinued. Although ADMV-043 was safely administered and well tolerated, protein expression did not meet a clinically meaningful level [http://investors.adverum.com/ news-releases/news-release-details/adverum-biotechnologiesprovides-program-updates]. On the other hand, a new study [60] shows that in a patient-derived fibroblasts, $\mathrm{C} 1-\mathrm{INH}$ secretion has been restored by vector administration of $\mathrm{C} 1-\mathrm{INH}$ gene, suggesting that it feasible to overcome the dominant negative disease mechanisms by gene supplementation. Nevertheless, Adverum announced that will not submit an Investigational New Drug application to FDA for ADVM-053 for the treatment of HAE by the end of 2018 .

\subsection{Antisense oligonucleotide (ASO) inhibiting prekallikrein}

Ionis Pharmaceuticals is developing IONIS-PKK-LRx, an antisense drug designed to reduce the production of prekallikrein (PKK), as a prophylactic treatment for patients with HAE.
A Phase 1 study in healthy volunteers has been completed. In this study, subjects treated with IONIS-PKKRx achieved dose-dependent reductions of up to $95 \%$ in PKK. Safety and tolerability profile of IONIS-PKKRx supports continued development. [Available from: http://www.ionispharma. com/pipeline/; https://www.bioportfolio.com/resources/trial/187317/ Safety-Tolerability-Pharmacokinetics-and-Pharmacodynamicsof-IONIS-PKK-LRx-Administered-Subcutaneously-to.html].

\section{Costs}

Chronically recurrent attacks of C1-INH-HAE cause a consistent humanistic and economic impact of the disease for patients and their caregivers. Long-term consequences for education attainment and careers have been reported in HAE patients [61-63]. On the other side, available for HAE are very expensive with costs per treated attack, in E.U. around 1500 euro and for prophylaxis up to 250,000 euro per patient per year. In U.S. costs are $4-5$ fold higher.

The perception that cost of treatment of rare diseases is inappropriately high compared to the total pharmaceutical expenditures and health-care costs is not completely correct. Based on an analysis conducted in the U.S. and E.U., the impact on costs is in line with the prevalence of these diseases in the population that is around $10 \%$. Expenditures on orphan drugs for orphan indications was approximately $\$ 33.5$ billion roughly reflecting the same proportion of pharmaceutical expenditures and only $1 \%$ of the total health-care expenditures [64].

The direct costs of treatment are considerably different worldwide. In several incoming countries the availability of new effective on demand and attack preventing treatments is often limited, nonetheless costs of not treating HAE appropriately are also quite high and difficult to estimate. It is hopeful that as the number of newly approved therapies to treat HAE will increase, the cost of therapy will decrease at the same time making treatment more accessible.

Trying to define the burden of the disease in the past years, patients were recruited from the US Hereditary Angioedema Association (HAEA) database [65]. Total annual per-patient costs were estimated at $\$ 42,000$ for the average HAE patient. The amount of indirect costs was estimated to be $\$ 16,000$ annually for the average patient considering the rates of missed work and loss of productivity.

A recent prospective observational study conducted in Italy over a period of one year in 2014 [66] reported total costs amounted to $€ 1.58$ million, equivalent to slightly more than $€ 11900$ per patient per year. The average cost for a single attack was $€ 1183$ (SD €789) including drug costs, emergency department visits, and diagnostic tests. The differences in the costs reported are explained by the different reimbursement modalities, even if differences in prescription of the drugs cannot be directly compared. However, these data are meant to get over in the next few years since the numbers (and costs) of new treatment are remarkable and their costeffectiveness is going to be evaluated as soon as possible based on real-life data.

Another important topic to be addressed regarding the evaluation of costs is the impact of health-care networking and organization. Spanò et al. [67] highlighted that an 
alternative and innovative treatment strategy, i.e. selfadministration, by focusing on treatment outcomes and costs, can create value for patients and health-care systems.

Despite cost savings, when the treatment is selfadministered or given in the home setting, in many countries treatment of attacks is only available in hospital and clinic settings.

\section{Unmet need for treatment of hereditary angioedema}

Frequency, location, and severity of angioedema recurrences in $\mathrm{HAE}$ patients remain unpredictable due to incomplete understanding of initial events leading to attacks. Thus, variability of clinical phenotypes remains obscure and adapting available treatments to such variability far from being perfect.

Current treatments are effective in minimizing the risk of death, but cannot guarantee disease control to all patients. Side effects and intravenous route of administration limit the use of LTP treatments that are diffusely available.

ODTs are on average very effective in reducing the length of the attacks, but not all patients can learn i.v. selfadministration and other present short-term relapse/recurrence of treated attacks.

High costs prevent access to treatment for several countries and question sustainability were available.

\section{Expert opinion}

The term hereditary angioedema refers to genetic diseases primarily characterized by recurrent angioedema that create risk of death due to asphyxia and temporary disability resulting in poor quality of life. Variants in four different genes can lead to HAE. For one form, C1-INH-HAE, the pathogenetic mechanism and the mediator are identified. When the mechanism of disease is known, treatments can be specifically targeted to block such mechanism.Current biological treatment options for HAE include plasma derived and recombinant $\mathrm{C} 1-\mathrm{INH}$, targeted therapies as icatibant and ecallantide but emerging treatment as monoclonal antibodies, small molecules and gene therapies are promising and near to be soon available.We prefer to consider 'biologic' not only therapies produced by living organisms, but also those that modify specific biologic conditions known to lead to a disease status. The era of recombinant DNA switched pharmacology from a biologic chemistry-based science to a biology-based science. The possibility to produce targeted antibodies demonstrated that it is possible to design a drug based on the action that we want to obtain. Thus, monoclonal antibody technique gave the first targeted biologic therapies and opened to the development of other biologic and non-biologic techniques to provide molecules with predetermined specific biologic activities.

In addition the development of gene therapy with SERPING1 supplementation is a new concept in the approach to the treatment of the disease. Nowadays, the treatment of C1-INH-HAE patients is based on an on-demand strategy, alone or in conjunction with long term prophylaxis. With the evolution of the gene therapy and, more generally biologic therapy, we can consider that the prophylaxis is extended to a very long period and, if effective, it is very close to reach a complete control of the disease and a great improvement in the quality of life of patients.

Looking at development of $\mathrm{C} 1-\mathrm{INH}-\mathrm{HAE}$, the disease mediator and the pathogenetic mechanism leading to its release was definitively proved in the 90 s of the last century, the same time that was facing the expansion of biotecks aimed at developing techniques for drug designing. With this in mind, we can understand why a disease with a population prevalence around 1:50,000 has on the market 6 biologic products made with different techniques, a seventh one on phase 3 and additional ones on early clinical/preclinical development. Obvious question is: is this a virtuous example of how health care should progress? Such an obvious question has no obvious answer. We know the costs of drug development, and we know that society pays for health-care costs. We state the question, but we are not going to give an answer. As physicians treating HAE we can see that the advent of biologic therapies dramatically improved the lives of our patients and that we still need further improvement. Indeed current treatments are effective in minimizing the risk of death, but cannot guarantee disease control to all patients. Whether this is cost/effective will be answered in the next 10 years when we will see if these major advances will benefit the majority of the patients.

\section{List of abbreviations}

HAE: hereditary angioedema

C1-INH: C1 inhibitor

FXII: Factor XII

HER2: human epidermal growth factor receptor 2

ODT: on demand treatment

LTP: $\quad$ long term prophylaxis treatment

STP: $\quad$ short term prophylaxis

BK B2R: bradykinin B2 receptor

pK: $\quad$ plasma kallikrein

FXIla: activated Factor XII

pd: $\quad$ plasma derived

FDA: food and drug administration

rh: recombinant human

mAb: monoclonal antibodies

ACE: angiotensin-converting enzyme

HK: high molecular weight kininogen

ADVM: adeno-associated virus vectors

A1AT: alpha-1 antitrypsin

ASO: $\quad$ antisense oligonucleotide

PKK: prekallikrein

U.S.: $\quad$ United States

E.U.: European Union

HAEA: US Hereditary Angioedema Association

\section{Funding}

This paper was not funded. 


\section{Declaration of interest}

M Cicardi and A Zanichelli have received meeting sponsorship from and performed clinical trial research funded by BioCryst, CSL Behring, Shire, Pharming Dyax Corporation. M Cicardi and A Zanichelli act as consultants for CSL Behring and Shire. F Perego has performed clinical research for, and reports receiving meeting sponsorship from, Shire and BioCryst. MA Wu has performed clinical research for, and reports receiving meeting sponsorship from, Shire and BioCryst. The authors have no other relevant affiliations or financial involvement with any organization or entity with a financial interest in or financial conflict with the subject matter or materials discussed in the manuscript apart from those disclosed.

\section{Reviewer Disclosures}

Peer reviewers on this manuscript have no relevant financial relationships or otherwise to disclose.

\section{References}

Papers of special note have been highlighted as either of interest $(\bullet)$ or of considerable interest (..) to readers.

1. Donaldson VH, Evans RR. A biochemical abnormality in herediatry angioneurotic edema: absence of serum inhibitor of $C^{\prime}$ 1-esterase. Am J Med. 1963.

2. Renne $T$. The procoagulant and proinflammatory plasma contact system. Semin Immunopathol. 2012;34(1):31-41.

3. Renne T, Schmaier AH, Nickel KF, et al. In vivo roles of factor XII. Blood. 2012;120(22):4296-4303.

4. Valerieva A, Perego F, Porebski G, et al. Hereditary angioedema: treatment options and availability. Balance between patients' needs and stakeholders' plans. Alergia Astma Immunologia. 2018;23(4):175-185.

5. Wu MA, Perego F, Zanichelli A, et al. Angioedema phenotypes: disease expression and classification. Clin Rev Allergy Immunol. 2016;51(2):162-169.

6. Vogelaar EF, Brummelhuis HG, Krijnen HW. Contributions to the optimal use of human blood. 3. Large-scale preparation of human C1 esterase inhibitor concentrate for clinical use. Vox Sang. 1974;26 (2):118-127.

7. Williams C, Wickerhauser M, Busbyet TF, et al. Pasteurization of C1 inactivator in the presence of citrate salts. Vox Sang. 1984;46 (5):260-269.

8. Soluk $L$, Price $H$, Sinclair $C$, et al. Pathogen safety of intravenous $R h$ immunoglobulin liquid and other immune globulin products: enhanced nanofiltration and manufacturing process overview. Am J Ther. 2008;15(5):435-443.

9. Cicardi M, Mannucci PM, Castelli R, et al. Reduction in transmission of hepatitis $C$ after the introduction of a heat-treatment step in the production of C1-inhibitor concentrate. Transfusion. 1995;35 (3):209-212.

10. Craig TJ, Rojavin MA, Machniget T, et al. Effect of time to treatment on response to $\mathrm{C} 1$ esterase inhibitor concentrate for hereditary angioedema attacks. Ann Allergy Asthma Immunol. 2013;111 (3):211-215

11. Zuraw BL, Busse PJ, White $\mathrm{M}$, et al. Nanofiltered $\mathrm{C} 1$ inhibitor concentrate for treatment of hereditary angioedema. N Engl J Med. 2010;363(6):513-522.

12. Zuraw BL, Kalfus I. Safety and efficacy of prophylactic nanofiltered C1-inhibitor in hereditary angioedema. Am J Med. 2012;125(9):938 e1-7.

13. Bernstein JA, Manning ME, Li H, et al. Escalating doses of $\mathrm{C} 1$ esterase inhibitor (CINRYZE) for prophylaxis in patients with hereditary angioedema. J Allergy Clin Immunol Pract. 2014;2(1):77-84.

14. Longhurst HJ, Carr S, Khair K. C1-inhibitor concentrate home therapy for hereditary angioedema: a viable, effective treatment option. Clin Exp Immunol. 2007;147(1):11-17.
15. Shapiro RS, Zacek L. Training hereditary angioedema patients to self-administer intravenous C1 esterase inhibitor concentrate. J Infus Nurs. 2014;37(4):284-290.

16. Zanichelli A, Azin GM, Cristina F, et al. Safety, effectiveness, and impact on quality of life of self-administration with plasma-derived nanofiltered $\mathrm{C} 1$ inhibitor (Berinert(R)) in patients with hereditary angioedema: the SABHA study. Orphanet J Rare Dis. 2018;13(1):51.

17. Caballero $T$, Farkas $H$, Bouillet $L$, et al. International consensus and practical guidelines on the gynecologic and obstetric management of female patients with hereditary angioedema caused by $\mathrm{C} 1$ inhibitor deficiency. J Allergy Clin Immunol. 2012;129(2):308-320.

18. Farkas $\mathrm{H}$, Kohalmi KV. Icatibant for the treatment of hereditary angioedema with C1-inhibitor deficiency in adolescents and in children aged over 2 years. Expert Rev Clin Immunol. 2018;14 (6):447-460.

- Evaluation of treatment in pediatric population.

19. Lumry W, Manning ME, Hurewitz DS, et al. Nanofiltered C1-esterase inhibitor for the acute management and prevention of hereditary angioedema attacks due to C1-inhibitor deficiency in children. J Pediatr. 2013;162(5):1017-22 e1-2.

- Evaluation of treatment in pediatric population.

20. Kreuz W, Rusicke E, Martinez-Saguer I, et al. Home therapy with intravenous human $\mathrm{C} 1$-inhibitor in children and adolescents with hereditary angioedema. Transfusion. 2012;52(1):100-107.

21. Aygoren-Pursun $E$, Soteres D, Moldovan $D$, et al. Preventing hereditary angioedema attacks in children using cinryze(R): interim efficacy and safety phase 3 findings. Int Arch Allergy Immunol. 2017;173(2):114-119.

22. Cicardi M, Zingale L. How do we treat patients with hereditary angioedema. Transfus Apher Sci. 2003;29(3):221-227.

23. Gandhi PK, Gentry WM, Bottorff MB. Thrombotic events associated with $\mathrm{C} 1$ esterase inhibitor products in patients with hereditary angioedema: investigation from the United States food and drug administration adverse event reporting system database. Pharmacotherapy. 2012;32(10):902-909.

24. Martinez-Saguer I, Cicardi M, Suffritti C, et al. Pharmacokinetics of plasma-derived $\mathrm{C} 1$-esterase inhibitor after subcutaneous versus intravenous administration in subjects with mild or moderate hereditary angioedema: the PASSION study. Transfusion. 2014;54 (6):1552-1561.

25. Zuraw BL, Cicardi M, Longhurst $\mathrm{HJ}$, et al. Phase II study results of a replacement therapy for hereditary angioedema with subcutaneous C1-inhibitor concentrate. Allergy. 2015;70(10):1319-1328.

26. Longhurst $\mathrm{H}$, Cicardi $M$, Craig $T$, et al. Prevention of hereditary angioedema attacks with a subcutaneous $\mathrm{C} 1$ inhibitor. $\mathrm{N}$ Engl J Med. 2017;376(12):1131-1140.

27. Haegarda-a subcutaneous $\mathrm{C} 1$ esterase inhibitor for prevention of hereditary angioedema. Med Lett Drugs Ther. 2018;60(1541):39-40.

28. Pawaskar D, Tortorici MA, Zuraw B, et al. Population pharmacokinetics of subcutaneous C1-inhibitor for prevention of attacks in patients with hereditary angioedema. Clin Exp Allergy. 2018;48 (10):1325-1332.

29. Zuraw B, Cicardi M, Levy MJ, et al. Recombinant human C1-inhibitor for the treatment of acute angioedema attacks in patients with hereditary angioedema. J Allergy Clin Immunol. 2010;126(4):821-827.e14.

30. Riedl MA, Bernstein JA, Li H, et al. Recombinant human C1-esterase inhibitor relieves symptoms of hereditary angioedema attacks: phase 3, randomized, placebo-controlled trial. Ann Allergy Asthma Immunol. 2014;112(2):163-169 e1.

31. Li H, Moldovan D, Bernstein JA, et al. Recombinant human-c1 inhibitor is effective and safe for repeat hereditary angioedema attacks. J Allergy Clin Immunol Pract. 2015;3(3):417-423.

32. Moldovan D, Reshef A, Fabiani J, et al. Efficacy and safety of recombinant human $\mathrm{C} 1$-inhibitor for the treatment of attacks of hereditary angioedema: european open-label extension study. Clin Exp Allergy. 2012;42(6):929-935.

33. Choi G, Soeters MR, Farkas $H$, et al. Recombinant human C1-inhibitor in the treatment of acute angioedema attacks. Transfusion. 2007;47(6):1028-1032. 
34. Riedl MA, Levy RJ, Suez D, et al. Efficacy and safety of recombinant C1 inhibitor for the treatment of hereditary angioedema attacks: a North American open-label study. Ann Allergy Asthma Immunol. 2013;110(4):295-299.

35. Reshef A, Moldovan D, Obtulowicz K, et al. Recombinant human C1 inhibitor for the prophylaxis of hereditary angioedema attacks: a pilot study. Allergy. 2013;68(1):118-124.

36. Riedl MA, Grivcheva-Panovska V, Moldovan D, et al. Recombinant human C1 esterase inhibitor for prophylaxis of hereditary angio-oedema: a phase 2, multicentre, randomised, double-blind, placebo-controlled crossover trial. Lancet. 2017;390(10102):1595-1602.

37. Farkas $\mathrm{H}$, Kohalmi KV, Veszeli $\mathrm{N}$, et al. Successful prophylaxis with recombinant human $\mathrm{C} 1$ inhibitor in a patient with hereditary angioedema. Ann Allergy Asthma Immunol. 2015;114(1):64-65.

38. Van Doorn MB, Burggraaf J, Van Dam T, et al. A phase I study of recombinant human $\mathrm{C} 1$ inhibitor in asymptomatic patients with hereditary angioedema. J Allergy Clin Immunol. 2005;116 (4):876-883.

39. Hack CE, Relan A, Baboeram A, et al. Immunosafety of recombinant human C1-inhibitor in hereditary angioedema: evaluation of ige antibodies. Clin Drug Investig. 2013;33(4):275-281.

40. Cicardi M, Levy RJ, McNeilet DL, et al. Ecallantide for the treatment of acute attacks in hereditary angioedema. N Engl J Med. 2010;363 (6):523-531.

41. Levy RJ, McNeil DL, Li HH, et al. EDEMA4: a phase 3, double-blind study of subcutaneous ecallantide treatment for acute attacks of hereditary angioedema. Ann Allergy Asthma Immunol. 2010;104(6):523-529.

42. Levy JH, O'Donnell PS. The therapeutic potential of a kallikrein inhibitor for treating hereditary angioedema. Expert Opin Investig Drugs. 2006;15(9):1077-1090.

43. Lehmann A. Ecallantide (Dyax/Genzyme). Curr Opin Invest Drugs. 2006;7(3):282-290.

44. Lumry WR, Bernstein JA, Li HH, et al. Efficacy and safety of ecallantide in treatment of recurrent attacks of hereditary angioedema: open-label continuation study. Allergy Asthma Proc. 2013;34 (2):155-161.

45. Chyung $Y$, Vince $B$, larrobino $R$, et al. A phase 1 study investigating DX-2930 in healthy subjects. Ann Allergy Asthma Immunol. 2014;113(4):460-6 e2.

46. Kenniston JA, Faucette RR, Martik D, et al. Inhibition of plasma kallikrein by a highly specific active site blocking antibody. J Biol Chem. 2014;289(34):23596-23608.

47. Banerji A, Busse $P$, Shennak $M$, et al. Inhibiting plasma kallikrein for hereditary angioedema prophylaxis. N Engl J Med. 2017;376 (8):717-728.

.. New drug with oral route od administration.

48. Banerji A, Riedl MA, Bernstein JA, et al. Effect of lanadelumab compared with placebo on prevention of hereditary angioedema attacks: a randomized clinical trial. JAMA. 2018;320(20):2108-2121.

.. New drug with oral route od administration.

49. Riedl MA, Bernstein JA, Craig T, et al. An open-label study to evaluate the long-term safety and efficacy of lanadelumab for prevention of attacks in hereditary angioedema: design of the HELP study extension. Clin Transl Allergy. 2017;7:36.

50. Bork K, Frank J, Grundt B, et al. Treatment of acute edema attacks in hereditary angioedema with a bradykinin receptor-2 antagonist (Icatibant). J Allergy Clin Immunol. 2007;119(6):1497-1503.
51. Cicardi M, Banerji A, Bracho F, et al. Icatibant, a new bradykinin-receptor antagonist, in hereditary angioedema. $\mathrm{N}$ Engl J Med. 2010;363(6):532-541.

- New drug with oral route od administration.

52. Malbran A, Riedl M, Ritchie B, et al. Repeat treatment of acute hereditary angioedema attacks with open-label icatibant in the FAST-1 trial. Clin Exp Immunol. 2014;177(2):544-553.

53. Lumry WR, Li HH, Levy RJ, et al. Randomized placebo-controlled trial of the bradykinin $\mathrm{B}(2)$ receptor antagonist icatibant for the treatment of acute attacks of hereditary angioedema: the FAST-3 trial. Ann Allergy Asthma Immunol. 2011;107(6):529-537.

54. Farkas $\mathrm{H}$, Avner R, Aberer W, et al. Treatment effect and safety of icatibant in pediatric patients with hereditary angioedema. J Allergy Clin Immunol Pract. 2017;1671-1678.e2.

55. Otani IM, Lumry WR, Hurwitz S, et al. Subcutaneous icatibant for the treatment of hereditary angioedema attacks: comparison of home self-administration with administration at a medical facility. J Allergy Clin Immunol Pract. 2017;5(2):442-447 e1.

56. Aygoren-Pursun E, Bygum A, Sci DM, et al. Oral plasma kallikrein inhibitor for prophylaxis in hereditary angioedema. $\mathrm{N}$ Engl J Med. 2018;379(4):352-362.

.- New drug with oral route od administration.

57. Qiu T, Chiuchiolo MJ, Whaley AS, et al. Gene therapy for C1 esterase inhibitor deficiency in a murine model of hereditary angioedema. Allergy. 2018.

58. Miesbach W, Meijer K, Coppens M, et al. Gene therapy with adeno-associated virus vector 5-human factor IX in adults with hemophilia B. Blood. 2018;131(9):1022-1031.

59. Rangarajan $S$, Walsh L, Lester W, et al. AAV5-factor VIII gene transfer in severe hemophilia A. N Engl J Med. 2017;377(26):2519-2530.

60. Haslund D, Ryø LB, Seidelin MS, et al. Dominant negative SERPING1 variants cause intracellular retention of $\mathrm{C} 1$-inhibitor in hereditary angioedema. J Clin Invest. 2018.

61. Aygoren-Pursun E, Bygum A, Beusterien K, et al. Socioeconomic burden of hereditary angioedema: results from the hereditary angioedema burden of illness study in Europe. Orphanet J Rare Dis. 2014;9:99.

62. Banerji A, Busse $P$, Christiansen SC, et al. Current state of hereditary angioedema management: a patient survey. Allergy Asthma Proc. 2015;36(3):213-217.

63. Lumry WR. Hereditary angioedema: the economics of treatment of an orphan disease. Front Med (Lausanne). 2018;5:22.

64. Divino V, DeKoven $M$, Kleinrock $M$, et al. Orphan drug expenditures in the United States: a historical and prospective analysis, 2007-18. Health Aff (Millwood). 2016;35(9):1588-1594.

65. Wilson DA, Bork K, Shea EP, et al. Economic costs associated with acute attacks and long-term management of hereditary angioedema. Ann Allergy Asthma Immunol. 2010;104(4):314-320.

66. Federici $C$, Perego F, Borsoi $L$, et al. Costs and effects of on-demand treatment of hereditary angioedema in Italy: a prospective cohort study of 167 patients. BMJ Open. 2018;8(7):e022291.

67. Spano R, Di Paola N, Bova M, et al. Value co-creation in healthcare: evidence from innovative therapeutic alternatives for hereditary angioedema. BMC Health Serv Res. 2018;18(1):571. 